\title{
Association Analysis of TEC Polymorphisms with Aspirin-Exacerbated Respiratory Disease in a Korean Population
}

\author{
Jin Sol Lee ${ }^{1,2 \dagger}$, Joon Seol Bae ${ }^{3 \dagger}$, Byung-Lae Park ${ }^{4}$, Hyun Sub Cheong ${ }^{4}$, Jeong-Hyun Kim ${ }^{1}$, \\ Jason Yongha Kim ${ }^{2}$, Suhg Namgoong ${ }^{2,4}$, Ji-On Kim ${ }^{2,4}$, Choon-Sik Park ${ }^{5 *}$, Hyoung Doo Shin ${ }^{2,4 * *}$ \\ ${ }^{1}$ Research Institute for Basic Science, Sogang University, Seoul 121-742, Korea, \\ ${ }^{2}$ Department of Life Science, Sogang University, Seoul 121-742, Korea, \\ ${ }^{3}$ Laboratory of Translational Genomics, Samsung Genome Institute, Samsung Medical Center, Seoul 135-710, Korea, \\ ${ }^{4}$ Department of Genetic Epidemiology, SNP Genetics, Inc., Seoul 153-801, Korea, \\ ${ }^{5}$ Division of Allergy and Respiratory Medicine, Soonchunhyang University Seoul Hospital, Seoul 140-743, Korea
}

The tyrosine-protein kinase Tec (TEC) is a member of non-receptor tyrosine kinases and has critical roles in cell signaling transmission, calcium mobilization, gene expression, and transformation. TEC is also involved in various immune responses, such as mast cell activation. Therefore, we hypothesized that TEC polymorphisms might be involved in aspirin-exacerbated respiratory disease (AERD) pathogenesis. We genotyped 38 TEC single nucleotide polymorphisms in a total of 592 subjects, which comprised 163 AERD cases and 429 aspirin-tolerant asthma controls. Logistic regression analysis was performed to examine the associations between TEC polymorphisms and the risk of AERD in a Korean population. The results revealed that TEC polymorphisms and major haplotypes were not associated with the risk of AERD. In another regression analysis for the fall rate of forced expiratory volume in 1 second $\left(\mathrm{FEV}_{1}\right)$ by aspirin provocation, two variations ( $r$ s 7664091 and $r$ s 12500534 ) and one haplotype (TEC_BL2 $h t 4)$ showed nominal associations with $\mathrm{FEV}_{1}$ decline $(p=0.03-0.04)$. However, the association signals were not retained after performing corrections for multiple testing. Despite TEC playing an important role in immune responses, the results from the present study suggest that TEC polymorphisms do not affect AERD susceptibility. Findings from the present study might contribute to the genetic etiology of AERD pathogenesis.

Keywords: aspirin-exacerbated respiratory disease, aspirin-tolerant asthma, Tec protein tyrosine kinase, genetic polymorphisms, haplotypes

\section{Introduction}

Asthma is a disease that is caused by inflammation in the lung and bronchus and is affected by genetic and environmental influences. Aspirin-exacerbated respiratory disease (AERD), which was first reported in 1922, is a type of asthma. AERD is characterized by the following three symptoms: bronchial asthma, aspirin sensitivity, and nasal polyposis [1-3]. It has been reported that $10-20 \%$ of asthma patients have aspirin sensitivity, whereas $1-2 \%$ of the non-asthma population shows aspirin sensitivity $[4,5]$. Although the mechanisms of AERD pathogenesis are still not fully understood, inflammatory responses by overproduction of leukotrienes are regarded as the main pathogenesis of AERD.

Aspirin is a commonly used medication, which belongs to the non-steroidal anti-inflammatory drugs. Despite being a widely used medication, aspirin intake also causes various side effects, including manifested gastrointestinal ulcer, stomach bleeding, and tinnitus, especially in higher doses. Although the side effects of aspirin are not common, these 
effects have been reported in about $10 \%$ of adult asthmatics. In AERD pathogenesis, aspirin inhibits the activation of cyclooxygenase-1 enzyme, leading to block of production of prostaglandin and thromboxane. This mechanism causes overproduction of leukotrienes, such as leukotriene B4, leukotriene $\mathrm{C} 4$, leukotriene $\mathrm{D} 4$, and leukotriene $\mathrm{E} 4[6,7]$.

The tyrosine-protein kinase Tec (TEC) is a member of the non-receptor tyrosine kinases and has critical roles in cell signaling transmission, calcium mobilization, gene expression, and transformation [8]. It has been known that TEC family kinases are associated with various intracellular signaling mechanisms, such as cytokine receptors and lymphocyte surface antigens $[9,10]$. In particular, it was demonstrated that the TEC family proteins are involved in regulation of leukotriene secretion via the mast cell signaling pathway [11]. Therefore, we hypothesized that TEC polymorphisms might be involved in AERD pathogenesis. In the present study, 38 TEC single nucleotide polymorphisms (SNPs) were genotyped in a total of 592 subjects, which comprised 163 AERD cases and 429 aspirin-tolerant asthma (ATA) controls, to examine the associations between TEC polymorphisms and AERD susceptibility.

\section{Methods}

\section{Study subjects}

Subjects in this study were recruited from the Asthma Genome Research Center, comprising the hospitals of Soonchunhyang, Chunnam, Chungbuk, Seoul National, and Chung-Ang Universities in Korea. All subjects provided written informed consents, and the study protocols were approved by the institutional review board of each hospital. Diagnosis of AERD was performed according to a modified method as previously described [12]. We also performed aspirin challenge in subjects with a history of aspirin hypersensitivity, presence of urticaria, nasal polyp, and sinusitis. The AERD case group included patients with $20 \%$ or greater decreases in forced expiratory volume in 1 second $\left(\mathrm{FEV}_{1}\right)$ or $15 \%$ to $19 \%$ decreases in $\mathrm{FEV}_{1}$ with naso-ocular or cutaneous reactions, whereas subjects showing a rate of $\mathrm{FEV}_{1}$ decline less than $15 \%$ without extrabronchial nasal or skin symptoms were included in the ATA group. The clinical diagnostic factors for the present study are summarized in Table 1.

\section{SNP selection and genotyping}

To investigate the associations between TEC polymorphisms and the risk of AERD, we selected candidate SNPs based on allele frequencies in the Asian population, linkage disequilibrium (LD) status, and National Center for Biotechnology information. The data for selection were obtained from the International HapMap database (http:// hapmap.ncbi.nlm.nih.gov/). Genotyping of 38 TEC polymorphisms was performed in a total of 592 subjects, including 163 AERD cases and 429 ATA controls. Genotyping was carried out with 20 ng of genomic DNA by TaqMan assay using $\mathrm{ABI}$ prism $7900 \mathrm{HT}$ sequence detection system software version 2.3 (Applied Biosystems, Foster City, CA, USA) in all subjects. Assay IDs of all SNPs used in TaqMan assay are listed in Supplementary Table 1.

Table 1. Clinical profile of subjects in study with TEC polymorphisms

\begin{tabular}{|c|c|c|c|c|}
\hline Clinical profile & Total subjects & AERD & ATA & p-value \\
\hline No. of subjects & 592 & 163 & 429 & \\
\hline Mean age of first medical examination (range, y) & $46.15(15.40-77.88)$ & $43.13(17.22-72.73)$ & $47.30(15.40-77.88)$ & 0.001 \\
\hline Sex (male/female) & $206 / 386$ & $59 / 104$ & $147 / 282$ & 0.66 \\
\hline Height $(\mathrm{cm})$ & $160.78 \pm 8.63$ & $161.72(143.00-196.00)$ & $160.42(140.00-199.00)$ & 0.10 \\
\hline Weight (kg) & $62.81 \pm 10.84$ & $61.25 \pm 10.38$ & $63.40 \pm 10.97$ & 0.03 \\
\hline BMI $\left(\mathrm{kg} / \mathrm{m}^{2}\right)$ & $24.24 \pm 3.39$ & $23.39 \pm 3.25$ & $24.58 \pm 3.39$ & 0.0001 \\
\hline Fall rate $(\%)$ & $9.27 \pm 13.24$ & $24.63 \pm 16.11$ & $3.54 \pm 4.85$ & 0.0001 \\
\hline FVC $\%$, predicted & $88.54 \pm 14.08$ & $90.35 \pm 14.04$ & $87.85 \pm 14.05$ & 0.05 \\
\hline $\mathrm{FEV}_{1} \%$, predicted & $90.54 \pm 16.97$ & $87.58 \pm 16.94$ & $91.66 \pm 16.87$ & 0.009 \\
\hline PC20, methacholine $(\mathrm{mg} / \mathrm{mL})$ & $6.43 \pm 8.67$ & $5.02 \pm 7.83$ & $6.91 \pm 8.90$ & 0.02 \\
\hline Current smoker $(\%)$ & 27.70 & 21.47 & 30.07 & 0.02 \\
\hline Blood eosinophil (\%) & $6.01 \pm 5.73$ & $5.96 \pm 5.21$ & $6.03 \pm 5.92$ & 0.88 \\
\hline Total IgE (IU/mL) & $357.65 \pm 604.09$ & $348.60 \pm 596.44$ & $361.00 \pm 607.56$ & 0.83 \\
\hline Positive rate of skin test (\%) & 56.42 & 52.76 & 57.81 & 0.27 \\
\hline
\end{tabular}

Age indicates first medical examination.

AERD, aspirin-exacerbated respiratory disease; ATA, aspirin-tolerant asthma; BMI, body mass index; FVC, forced vital capacity; FEV ${ }_{1}$, forced expiratory volume in 1 second. 


\section{Statistics}

We applied a widely used measure of linkage disequilibrium to all pairs of biallelic loci: Lewontin's D' (|D'|) [13] and $r^{2}$. Haplotypes of each individual were inferred using the PHASE algorithm (ver. 2.0), developed by Stephens et al. [14]. Linear regression analysis was performed to examine the differences in the rates of decline in $\mathrm{FEV}_{1}$ following aspirin challenge among the genotypes and major haplotypes. The data were managed and analyzed using Statistical Analysis System, version 9.2 (SAS Inc., Cary, NC, USA). Associations for AERD under the logistic model were adjusted by smoking status, atopy, body mass index (BMI), age, and sex (male $=0$, female $=1$ ). Significant associations are shown in boldface $(\mathrm{p}<0.05)$.

\section{Results and Discussion}

We recruited a total of 592 subjects, which consisted of 163 AERD cases and 429 ATA controls, for the present study. According to the results, four clinical characteristics showed significant differences between the case and control groups (Table 1). The fall rate by aspirin provocation was signifi- cantly higher in AERD subjects than ATA controls (24.63 \pm 16.11 vs. $3.54 \pm 4.85 ; \mathrm{p}=0.0001)$. Percentage of predicted $\mathrm{FEV}_{1}$ in the AERD subjects showed decreased lung function than in ATA subjects $(87.58 \pm 16.94$ vs. $91.66 \pm 16.87$; $\mathrm{p}=$ 0.009). Also, age of first medical examination and BMI were lower in AERD cases than ATA controls. The other diagnostic factors showed no significant differences between the case and control group.

In the present study, 38 TEC polymorphisms and 12 major haplotypes were used for the association analyses. Locations of the polymorphisms are shown in a genetic map of TEC with their LD status (Fig. 1A). Minor allele frequencies (MAFs) of the SNPs in Korean subjects are displayed in Supplementary Table 2 with their allele change, position, and p-value for Hardy-Weinberg equilibrium. All SNPs were in Hardy-Weinberg equilibrium. The LD blocks were obtained by using HaploView software, and the haplotypes of each LD block were calculated by PHASE software (Fig. 1B and 1C). We used major haplotypes that had frequencies higher than 0.05 for further analyses. The minor haplotypes that had frequencies lower than 0.05 were merged and presented as 'Others.' To compare genetic differences among ethnicities, we obtained MAFs of Caucasians, Han Chinese,

(A)

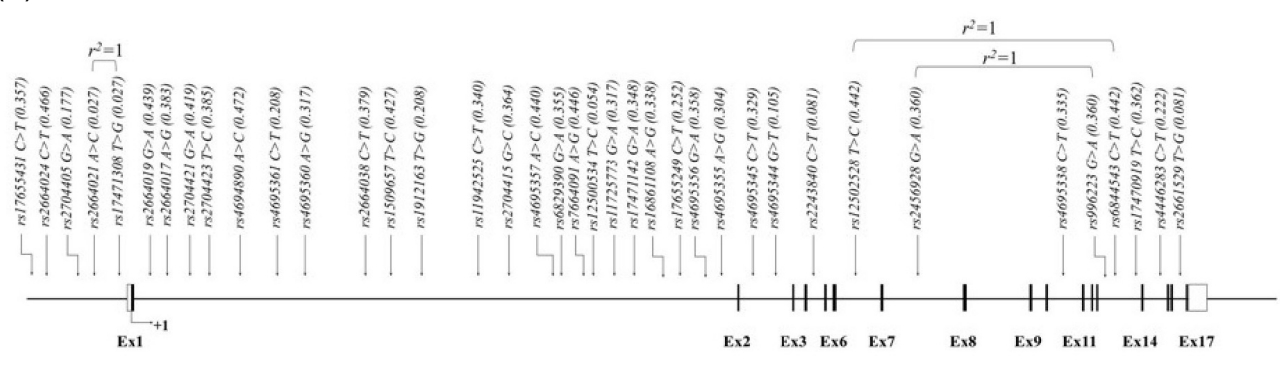

(B)

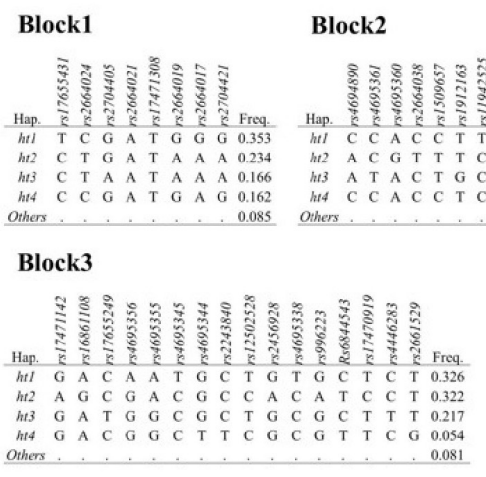

(C)

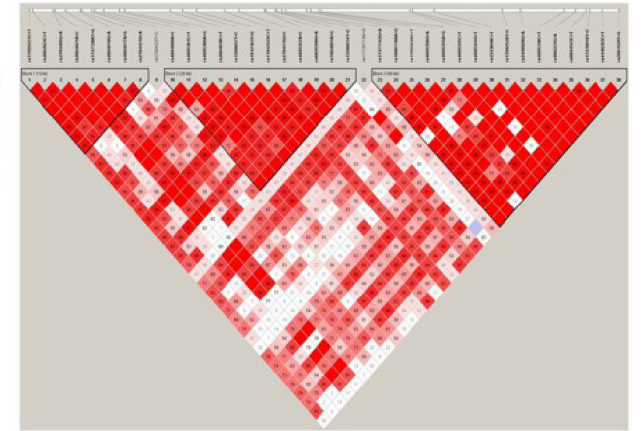

Fig. 1. Schematic physical map, haplotypes and linkage disequilibrium (LD) plot of TEC. (A) Polymorphisms identified in TEC. Coding exons are marked by shaded blocks and untranslated region by white blocks. The LD coefficients $\left(r^{2}\right)$ are based on the genotypes of Korean samples. (B) Haplotypes are calculated using genotypes of TEC polymorphisms in a Korean population. Only those with frequencies over 0.05 are shown in tables. Haplotypes with frequencies lower than 0.05 are merged into "Others." (C) LD coefficients (|D'|) among the selected SNPs based on the genotypes of whole study subjects in this study. 
Table 2. Association analysis of TEC polymorphisms and haplotypes with the risk of AERD in a Korean population

\begin{tabular}{|c|c|c|c|c|c|}
\hline SNP/Haplotype & Allele change & Position & OR $(95 \% \mathrm{Cl})$ & $\mathrm{p}$-value & Statistical power $(\%)$ \\
\hline rs17655431 & $C>T$ & Promoter & $1.11(0.85-1.45)$ & 0.43 & 80.33 \\
\hline rs2664024 & $C>T$ & Promoter & $0.98(0.75-1.27)$ & 0.87 & 82.29 \\
\hline rs2704405 & $G>A$ & Promoter & $1.03(0.73-1.44)$ & 0.87 & 56.53 \\
\hline rs2664021 & $A>C$ & Promoter & $0.82(0.34-1.98)$ & 0.66 & 4.53 \\
\hline rs17471308 & $\mathrm{T}>\mathrm{G}$ & Promoter & $0.82(0.34-1.98)$ & 0.66 & 4.53 \\
\hline rs2664019 & $G>A$ & Intron 1 & $0.99(0.76-1.30)$ & 0.97 & 82.32 \\
\hline rs2664017 & $A>G$ & Intron 1 & $1.10(0.84-1.44)$ & 0.48 & 81.33 \\
\hline$r s 2704421$ & $G>A$ & Intron 1 & $1.03(0.79-1.35)$ & 0.81 & 82.14 \\
\hline rs2704423 & $\mathrm{T}>\mathrm{C}$ & Intron 1 & $1.02(0.79-1.33)$ & 0.86 & 81.39 \\
\hline TEC_BL1_ht1 & - & - & $1.11(0.85-1.45)$ & 0.46 & 80.09 \\
\hline TEC_BL1_ht2 & - & - & $1.05(0.77-1.43)$ & 0.74 & 68.14 \\
\hline TEC_BL1_ht3 & - & - & $0.98(0.69-1.39)$ & 0.91 & 54.38 \\
\hline TEC_BL1_ht4 & - & - & $0.84(0.59-1.22)$ & 0.36 & 52.40 \\
\hline rs4694890 & $A>C$ & Intron 1 & $1.06(0.82-1.38)$ & 0.64 & 82.24 \\
\hline rs4695361 & $\mathrm{C}>\mathrm{T}$ & Intron 1 & $0.91(0.66-1.25)$ & 0.56 & 63.71 \\
\hline rs4695360 & $A>G$ & Intron 1 & $1.01(0.77-1.34)$ & 0.93 & 77.96 \\
\hline rs 2664038 & $\mathrm{C}>\mathrm{T}$ & Intron 1 & $1.06(0.82-1.37)$ & 0.68 & 81.20 \\
\hline rs1509657 & $\mathrm{T}>\mathrm{C}$ & Intron 1 & $0.97(0.74-1.27)$ & 0.83 & 82.23 \\
\hline rs1912163 & $\mathrm{T}>\mathrm{G}$ & Intron 1 & $0.88(0.64-1.21)$ & 0.43 & 63.71 \\
\hline rs11942525 & $\mathrm{C}>\mathrm{T}$ & Intron 1 & $1.09(0.83-1.43)$ & 0.55 & 79.46 \\
\hline rs 2704415 & $G>C$ & Intron 1 & $1.13(0.86-1.48)$ & 0.37 & 80.64 \\
\hline rs4695357 & $A>C$ & Intron 1 & $0.92(0.71-1.21)$ & 0.55 & 82.32 \\
\hline rs6829390 & $G>A$ & Intron 1 & $1.05(0.80-1.38)$ & 0.74 & 80.24 \\
\hline rs7664091 & $A>G$ & Intron 1 & $0.94(0.71-1.22)$ & 0.62 & 82.34 \\
\hline rs12500534 & $\mathrm{T}>\mathrm{C}$ & Intron 1 & $0.63(0.33-1.20)$ & 0.16 & 12.90 \\
\hline rs 11725773 & $G>A$ & Intron 1 & $1.16(0.89-1.52)$ & 0.27 & 77.96 \\
\hline TEC_BL2_ht1 & - & - & $1.04(0.79-1.37)$ & 0.79 & 79.10 \\
\hline TEC_BL2_ht2 & - & - & $1.03(0.78-1.37)$ & 0.83 & 76.58 \\
\hline TEC_BL2_ht3 & - & - & $0.82(0.59-1.15)$ & 0.25 & 61.80 \\
\hline TEC_BL2_ht4 & - & - & $0.63(0.33-1.20)$ & 0.16 & 12.90 \\
\hline rs17471142 & $G>A$ & Intron 1 & $1.18(0.89-1.55)$ & 0.25 & 79.89 \\
\hline rs16861108 & $A>G$ & Intron 1 & $1.23(0.94-1.63)$ & 0.14 & 79.34 \\
\hline rs17655249 & $C>T$ & Intron 1 & $0.73(0.53-1.00)$ & 0.05 & 71.20 \\
\hline rs4695356 & $G>A$ & Intron 1 & $1.00(0.76-1.32)$ & 1.00 & 80.38 \\
\hline rs4695355 & $A>G$ & Intron 1 & $0.80(0.60-1.07)$ & 0.13 & 76.93 \\
\hline rs4695345 & $C>T$ & Intron 2 & $1.05(0.80-1.38)$ & 0.73 & 78.79 \\
\hline rs4695344 & $G>T$ & Intron 2 & $0.80(0.52-1.23)$ & 0.31 & 32.83 \\
\hline rs 2243840 & $\mathrm{C}>\mathrm{T}$ & Intron 4 & $0.91(0.56-1.48)$ & 0.70 & 23.31 \\
\hline rs12502528 & $\mathrm{T}>\mathrm{C}$ & Intron 7 & $1.13(0.86-1.48)$ & 0.39 & 82.33 \\
\hline rs2456928 & $G>A$ & Intron 8 & $1.17(0.89-1.54)$ & 0.26 & 80.47 \\
\hline rs4695338 & $\mathrm{C}>\mathrm{T}$ & Intron 10 & $1.05(0.80-1.39)$ & 0.71 & 79.16 \\
\hline rs996223 & $G>A$ & Intron 13 & $1.17(0.89-1.54)$ & 0.26 & 80.47 \\
\hline rs6844543 & $\mathrm{C}>\mathrm{T}$ & Intron 13 & $1.13(0.86-1.48)$ & 0.39 & 82.33 \\
\hline rs17470919 & $\mathrm{T}>\mathrm{C}$ & Intron 13 & $1.15\left(0.88^{-1.52)}\right.$ & 0.31 & 80.55 \\
\hline rs4446283 & $\mathrm{C}>\mathrm{T}$ & Intron 14 & $0.80(0.58-1.11)$ & 0.18 & 66.41 \\
\hline rs2661529 & $\mathrm{T}>\mathrm{G}$ & Intron 16 & $0.90(0.55-1.46)$ & 0.66 & 23.31 \\
\hline TEC_BL3_ht1 & - & - & $1.07(0.81-1.42)$ & 0.62 & 78.59 \\
\hline TEC_BL3_ht2 & - & - & $1.26(0.95-1.66)$ & 0.11 & 78.25 \\
\hline TEC_BL3_ht3 & - & - & $0.80(0.58-1.12)$ & 0.19 & 65.85 \\
\hline TEC_BL3_ht4 & - & - & $1.22(0.71-2.12)$ & 0.47 & 12.18 \\
\hline
\end{tabular}

Logistic analyses controlling for age, sex, smoking status, atopy, and body mass index as covariates, performed using the Statistical Analysis System (SAS).

AERD, aspirin-exacerbated respiratory disease; SNP, single nucleotide polymorphism; OR, odds ratio; Cl, confidence interval. 
Japanese, and Africans from the NCBI database (dbSNP) (Supplementary Table 2) and calculated LD blocks using genotype data from the International HapMap database (Supplementary Fig. 1). As a result, Asian populations showed similar MAFs, whereas the other populations showed distinct differences. However, we did not find any correlation in LD status in any ethnicity.

Logistic analyses were performed to investigate the associations between TEC polymorphisms and the risk of AERD. The result from analyses revealed that all SNPs and major haplotypes were not associated with the risk of AERD (Table 2). On the other hand, two SNPs ( $r$ 7664091, $\mathrm{p}=0.04$ and rs 12500534, $\mathrm{p}=0.03$ ) and one major haplotype in LD block 2 (TEC_BL2_ht4, $\mathrm{p}=0.03$ ) showed marginal associations in the regression analysis with the decline rate of $\mathrm{FEV}_{1}$ (Supplementary Table 3). However, the association signals of these polymorphisms disappeared after performing corrections for multiple testing (data not shown). Taken together, these results indicate that the TEC polymorphisms are not associated with AERD susceptibility, at least in the Korean population. The lack of associations in this study suggests that although TEC plays an important role in immune responses, TEC variants do not directly affect decreased pulmonary function by aspirin uptake. However, considering the fact that the difference in frequency of polymorphisms showed different effects in various populations, replication studies in AERD subjects in other ethnicities are recommended.

The present study still had several limitations. First, an average statistical power of $66.41 \%$ indicated an insufficient sample size. However, the rare condition of AERD made it difficult to recruit subjects in the Korean asthma cohort. Second, SNPs in coding sequence were not selected, due to very low frequencies, although non-synonymous SNPs (nsSNP) in exonic regions could affect risk of the disease. Further studies are required to examine the molecular role of nsSNPs in TEC.

In conclusion, we hypothesized that TEC might impact on AERD susceptibility. However, our results showed that 38 TEC polymorphisms and 12 major haplotypes were not associated with the risk of AERD. Although further studies are required to investigate the exact role of the TEC SNPs in immune responses, the preliminary results of the present study may provide useful information for AERD pathogenesis.

\section{Supplementary materials}

Supplementary data including three tables and one figure can be found with this article online at http://www.geno- minfo.org/src/sm/gni-12-58-s001.pdf.

\section{Acknowledgments}

This work was supported by a grant from the Korea Health 21 R\&D Project (A010249). This work was supported by a grant from the Priority Research Centers Program through the National Research Foundation of Korea (NRF), funded by the Ministry of Education, Science and Technology (2012-0006690). The DNA samples were generously provided by Soonchunhyang University, Bucheon Hospital Biobank, and a member of the National Biobank of Korea, supported by the Ministry of Health, Welfare and Family Affairs, Republic of Korea.

\section{References}

1. Kim SH, Ye YM, Hur GY, Lee SK, Sampson AP, Lee HY, et al. CysLTR1 promoter polymorphism and requirement for leukotriene receptor antagonist in aspirin-intolerant asthma patients. Pharmacogenomics 2007;8:1143-1150.

2. Kim SH, Kim YK, Park HW, Kim SH, Kim SH, Ye YM, et al. Adenosine deaminase and adenosine receptor polymorphisms in aspirin-intolerant asthma. Respir Med 2009;103: 356-363.

3. Palikhe NS, Kim SH, Park HS. What do we know about the genetics of aspirin intolerance? J Clin Pharm Ther 2008;33: 465-472.

4. Szczeklik A, Nizankowska E, Duplaga M. Natural history of aspirin-induced asthma. AIANE Investigators. European Network on Aspirin-Induced Asthma. Eur Respir J 2000;16: 432-436.

5. Hedman J, Kaprio J, Poussa T, Nieminen MM. Prevalence of asthma, aspirin intolerance, nasal polyposis and chronic obstructive pulmonary disease in a population-based study. Int $J$ Epidemiol 1999;28:717-722.

6. Funk CD. Prostaglandins and leukotrienes: advances in eicosanoid biology. Science 2001;294:1871-1875.

7. Chang WC, Nelson C, Parekh AB. Ca2+ influx through CRAC channels activates cytosolic phospholipase A2, leukotriene C4 secretion, and expression of c-fos through ERK-dependent and -independent pathways in mast cells. FASEB J 2006;20: 2381-2383.

8. Sato K, Mano H, Ariyama T, Inazawa J, Yazaki Y, Hirai H. Molecular cloning and analysis of the human Tec protein-tyrosine kinase. Leukemia 1994;8:1663-1672.

9. Desiderio S. Human genetics: becoming B cells. Nature 1993; 361:202-203.

10. Qiu Y, Kung HJ. Signaling network of the Btk family kinases. Oncogene 2000;19:5651-5661.

11. Schmidt U, Abramova A, Boucheron N, Eckelhart E, Schebesta A, Bilic I, et al. The protein tyrosine kinase Tec regulates mast cell function. Eur J Immunol 2009;39:3228-3238. 
12. Park HS. Early and late onset asthmatic responses following lysine-aspirin inhalation in aspirin-sensitive asthmatic patients. Clin Exp Allergy 1995;25:38-40.

13. Hedrick PW. Gametic disequilibrium measures: proceed with caution. Genetics 1987;117:331-341.

14. Stephens M, Smith NJ, Donnelly P. A new statistical method for haplotype reconstruction from population data. Am J Hum Genet 2001;68:978-989. 